\title{
Screening and Confirmatory Anti-Neutrophil Cytoplasmic Antibodies (ANCA) Testing for Rapidly Progressive Glomerulonephritis (RPGN): A Tertiary Care Experience
}

\author{
Dr. Faisal Ansari ${ }^{1^{*}}$, Dr. Ram Mohan ${ }^{1}$, Dr. Vijay Dharma Teja ${ }^{1}$ \\ ${ }^{1}$ Department of Microbilogy, Nizams Institute of Medical Sciences, Punjagutta Rd, Punjagutta Market, Punjagutta, Hyderabad, \\ Telangana 500082, India
}

DOI: $10.36348 /$ sipm.2021.v06i03.002

| Received: 08.10.2019 | Accepted: 19.10.2019| Published: 15.03.2021

*Corresponding author: Dr. Faisal Ansari

\section{Abstract}

Objective: Simultanaeous testing for serum antineutrophil cytoplasmic antibodies (ANCA) by indirect immunofluorescence (IF) and by anti-proteinase-3 (PR3)/anti-myeloperoxidase (MPO) antibody assays may identify patients with PR3-ANCA or MPO-ANCA despite a negative IF (IF negative MPO/PR3-positive); however, the significance of this result is not clear. We sought to determine whether IF-negative MPO/PR3-positive results identified any cases of clinically meaningful systemic vasculitis at our institution. Methods: We conducted a retrospective analysis of all ANCA positive RPGN patients either by IF or ELISA identified at our institution from August 2017 - July 2018. Results: Out of 265 samples 45 were positive for both IF and ELISA, 220 were IF-negative. Among IF negative, 6 samples $(2.7 \%)$, tested positive for MPO-ANCA or PR3-ANCA. Two IF-negative ELISA positive patients were subsequently diagnosed with ANCA-associated renal limited vasculitis. Two IF-negative ELISA-positive patients were previously diagnosed and treated for AAV, both with positive IF and antibody tests prior to treatment. 1 patient had SLE


$33 \%$ only 1 pt had both C- ANCA AND P-ANCA patterns positive. Conclusion: In our study both IF and ELISA, ELISA positivity without a positive IF rarely led to a definite diagnosis of systemic vasculitis, and was more likely to occur in the context of a non-vasculitis inflammatory condition. Our results suggest that concurrent IF and MPO/PR3 testing may be important in preventing a missed diagnosis of new onset renal limited AAV.

Keywords: Antineutrophil cytoplasmic antibodies (ANCA) vasculitis Rapidly Progressive Glomerulonephritis (RPGN), outcomes.

Copyright (C) 2021 The Author(s): This is an open-access article distributed under the terms of the Creative Commons Attribution 4.0 International License (CC BY-NC 4.0) which permits unrestricted use, distribution, and reproduction in any medium for non-commercial use provided the original author and source are credited.

\section{INTRODUCTION}

Among ANCA associated vasculitis (AAV). $60 \%$ of the RPGN are ANCA associated. Indirect immunofluorescence performed followed by confirmation with (ELISAs). Testing for serum (ANCA) by IF and ELISA may identify patients with PR3-ANCA or MPO-ANCA despite a negative IF however, the significance of this result is not clear [1, 2]. We determine whether IF-negative, ELISA-positive results in systemic or renal limited ANCA associated vasculitis (AAV) in cases of RPGN at our institution. PR3-ANCA can also serve as an aid for the diffrentiation between ulcerative colitis (UC) and Crohn's disease $(\mathrm{CrD})$ and the stratification of UC patients $[3,4]$.
Comparisons of the utility of IF and specific antibody assays for the diagnosis of AAV have frequently, though not uniformly, suggested that IF is more sensitive than MPO-ANCA and PR3-ANCA assays [3-7]. However, because of the low specificity of P-ANCA and CANCA IF patterns for AAV, MPO/PR3-ANCA assays may provide a better positive predictive value and likelihood ratio compared to IF, while the combination of the 2 demonstrates the best results $[4,8]$.

The International Consensus Statement on the testing and reporting of ANCAs recommended that all samples sent for diagnostic ANCA testing be evaluated by IF, and that samples with cytoplasmic fluorescence, or nuclear fluorescence in a homogenous or peripheral 
nuclear pattern, be subsequently tested for MPO-ANCA and PR3-ANCA [9]. The consensus statement also states that optimally, all serum samples should be tested for MPO-ANCA and PR3-ANCA. One common clinical approach is to screen all serum samples by IF, and then test only IF-positive samples for MPO-ANCA and PR3-ANCA. Alternatively, some practitioners utilize specific antibody tests first, followed by reflex IF testing only on MPO/PR3-ANCA-positive samples [7, $10]$, and in some specific cases, MPO/PR3-ANCA tests may be used alone [11].

Testing samples by IF and specific antibody tests simultaneously can identify IF-negative MPO/PR3-positive patients who would be otherwise missed if only IF-positive samples were tested for MPO/PR3-ANCA. Prior reports have noted a small number of such IF-MPO/ PR3-positivesamples [4, 12]; however, the clinical significance of this result is unclear, as MPO/PR3-ANCA can also sometimes be detected in non-vasculitic conditions, including SLE and IBD $[13,14]$.

\section{MATERIALS AND METHODS}

This study was done at Nizams Institute of Medical sciences. Results of all ANCA tests ordered through NIMS from August 2017 - July 2018 were collected. These samples come from inpatients and outpatients at NIMS and patients seen at associated outpatient centers. Through this period, all samples sent for evaluation for serum ANCA as part of clinical care, including for the evaluation of clinically suspected systemic vasculitis, monitoring of vasculitis disease activity, or any other indication, were included. All samples were evaluated by both IF and multiplex bead assays for MPO-ANCA and PR3-ANCA as part of routine clinical laboratory practice.

IF was performed by the Nizams Institute of Medical sciences laboratory. Serum samples were diluted 1:20 and incubated on ethanol-fixed human neutrophil substrate slides as per the manufacturer's method recommendations (Euroimmun kit, Lubeck Germany). IF patterns were reported as cytoplasmic, perinuclear, atypical, or negative. Only cytoplasmic or perinuclear patterns were considered positive in this study. Atypical results were excluded from analyses. MPO-ANCA and PR3-ANCA were measured by multiplexed bead assays, a type of solid phase immunoassay, performed at a commercial laboratory (ARUP Laboratories, Salt Lake City, UT). An antibody level $>25$ units $/ \mathrm{mL}$ was considered positive. Previous analyses demonstrated very good concordance between results obtained on split samples, over a broad range of levels, analyzed with this multiplexed bead assay, with another commercial ELISA kit Euroimmun kit, Lubeck Germany )and with assays performed at Nizams Institute of Medical sciences laboratory
Statistical analysis: The data calculated from study were subjected to simple descriptive statistical analysis using percentage

\section{RESULTS}

Out of 265 samples 45 were positive for both IF and ELISA, 220 were IF-negative. Among IF negative, 6 samples $(2.7 \%)$, tested positive for MPOANCA or PR3-ANCA. Two IF-negative ELISA positive patients were subsequently diagnosed with ANCA-associated renal limited vasculitis. Two IFnegative ELISA-positive patients were previously diagnosed and treated for AAV, both with positive IF and antibody tests prior to treatment. 1 patient had SLE and 1 had inflammatory bowel disease. Mean age of

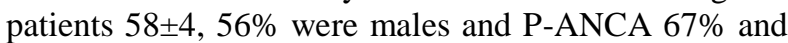
C-ANCA $33 \%$ only 1 pt had both C- ANCA AND PANCA patterns positive

\section{DISCUSSION}

Testing for the presence of ANCA is a key component of the diagnostic evaluation for AAV; however, significant variability exists in the strategies employed to detect ANCA, including reflex testing (test by IF first, and if positive, test for MPO-ANCA and PR3-ANCA) and concurrent testing (test by IF and MPO/PR3-ANCA assays simultaneously). It is well established that isolated IF positivity, without an associated MPO-ANCA or PR3-ANCA, has a poor predictive value for AAV [4]; however, the significance of positive MPO-ANCA or PR3-ANCA in the absence of IF positivity is less clear.

In this report of over 1 year of concurrent ANCA testing by IF and MPO/PR3 multiplexed bead assays at NIMS Out of 265 samples 45 were positive for both IF and ELISA, 220 were IF-negative Among IF negative, 6 samples $(2.7 \%)$, tested positive for MPOANCA or PR3-ANCA. Our study is unique as two IFnegative ELISA positive patients were subsequently diagnosed with ANCA-associated renal limited vasculitis. Two IF-negative ELISA-positive patients were previously diagnosed and treated for AAV, both with positive IF and antibody tests prior to treatment. 1 patient had SLE and 1 had inflammatory bowel disease. $56 \%$ were males and P-ANCA $67 \%$ and C-ANCA $33 \%$ only 1 pt had both C- ANCA AND P-ANCA patterns positive

The frequency of this result will vary with the specifics of test methods $[3,6,16,17]$, but is within the range of prior reports $[4,12,18]$. IF-negative MPO/PR3-positive results have been previously described in patients with conditions including inflammatory arthritis, IBD, connective tissue disease $[7,8,12,19]$; however, there is little data reported on significance of this result in routine clinical testing (4, 12). Stone et al noted 5 IF-negative MPO/PR3-positive patients out of 856 consecutive patients evaluated for 
AAV, but the clinical associations were not described [4]. Tsiveriotis reported 17 IF-negative MPO/PR3positive patients out of 4786 outpatients concurrently tested, including 2 with connective tissue diseases, 4 with mixed/undefined disorders, and 11 with AAV; however, new diagnosis of AAV was not distinguished from previously treated AAV [12].

It has been suggested that MPO/PR3-ANCA testing alone may be reasonable in emergent clinical situations [11], indicating that caution must be used when interpreting positive MPO/ PR3-ANCA results in the absence of concurrent IF testing. Consistent with previous reports $[5,12,13]$, we identified patients with SLE, IBD, and other inflammatory disorders with IFnegative MPO/PR3-positive results. This phenomenon may be due to a number of mechanisms, including antibody cross-reactivity in the multiplex bead assay, higher sensitivity of the multiplex bead assay, specific characteristics of the antigenic epitopes targeted, or technical variability $[7,11]$.

In conclusion, In our study both IF and ELISA, ELISA positivity without a positive IF rarely led to a definite diagnosis of systemic vasculitis, and was more likely to occur in the context of a nonvasculitis inflammatory condition. Our results suggest that concurrent IF and MPO/PR3 testing may be important in preventing a missed diagnosis of new onset renal limited AAV.


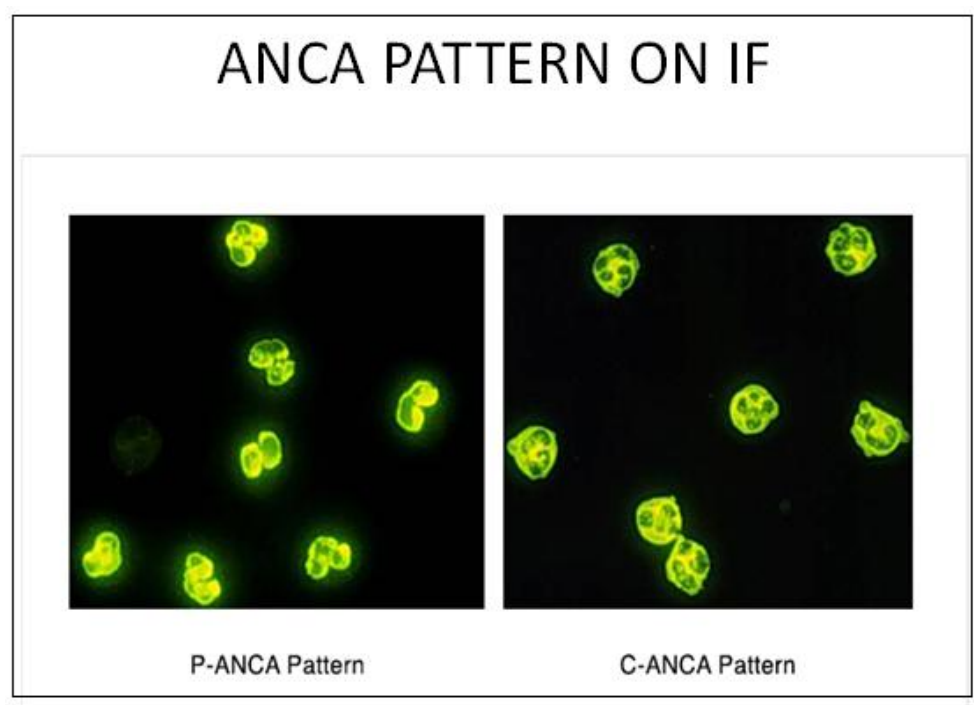




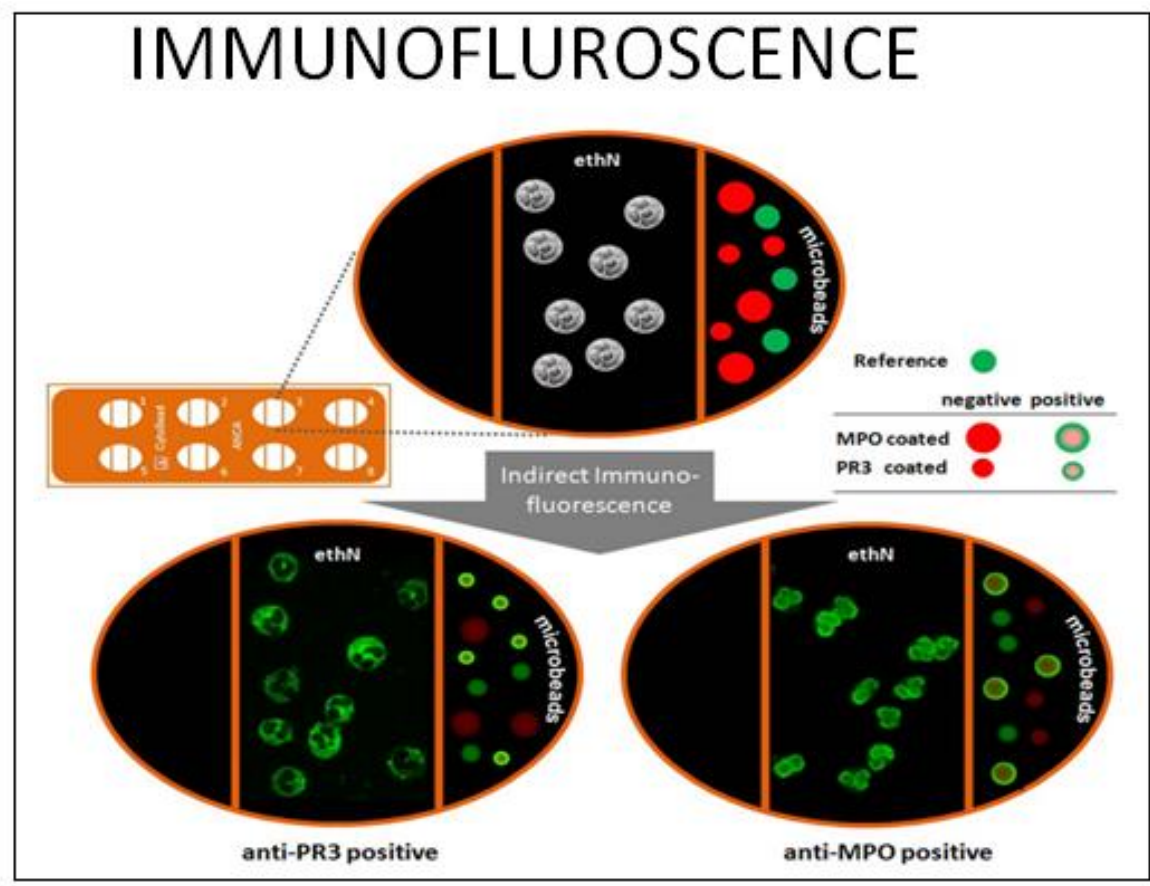

\section{Conclusion}

In our study both IF and ELISA, ELISA positivity without a positive IF rarely led to a definite diagnosis of systemic vasculitis, and was more likely to occur in the context of a non-vasculitis inflammatory condition. Our results suggest that concurrent IF and MPO/PR3 testing may be important in preventing a missed diagnosis of new onset renal limited AAV.

\section{REFERENCES}

1. Rao, D. A., Wei, K., Merola, J. F., O’Brien, W. R., Takvorian, S. U., Dellaripa, P. F., \& Schur, P. H. (2015). The significance of MPO-ANCA and PR3-ANCA without immunofluorescent ANCA found by routine clinical testing. The Journal of rheumatology, 42(5), 847-852.

2. Sowa, M., Grossmann, K., Knütter, I., Hiemann, R., Röber, N., Anderer, U., ... \& Schierack, P. (2014). Simultaneous automated screening and confirmatory testing for vasculitis-specific ANCA. PLoS One, 9(9), e107743.

3. Schulte-Pelkum, J., Radice, A., Norman, G. L., López Hoyos, M., Lakos, G., Buchner, C., ... \& Mahler, M. (2014). Novel clinical and diagnostic aspects of antineutrophil cytoplasmic antibodies. Journal of immunology research, 2014.

4. Talor, M. V., Stone, J. H., Stebbing, J., Barin, J., Rose, N. R., \& Burek, C. L. (2007). Antibodies to selected minor target antigens in patients with anti- neutrophil cytoplasmic antibodies (ANCA). Clinical \& Experimental Immunology, 150(1), 42-48.

5. Trevisin, M., Pollock, W., Dimech, W., \& Savige, J. (2008). Evaluation of a multiplex flow cytometric immunoassay to detect PR3-and MPOANCA in active and treated vasculitis, and in inflammatory bowel disease (IBD). Journal of immunological methods, 336(2), 104-112.

6. Csernok, E., Holle, J., Hellmich, B., Willem, J., Tervaert, C., Kallenberg, C. G., ... \& Gross, W. L. (2004). Evaluation of capture ELISA for detection of antineutrophil cytoplasmic antibodies directed against proteinase 3 in Wegener's granulomatosis: first results from a multicentre study. Rheumatology, 43(2), 174-180.

7. Csernok, E., \& Moosig, F. (2014). Current and emerging techniques for ANCA detection in vasculitis. Nature Reviews Rheumatology, 10(8), 494.

8. Holle, J. U., Csernok, E., Fredenhagen, G., Backes, M., Bremer, J. P., \& Gross, W. L. (2010). Clinical evaluation of hsPR3-ANCA ELISA for detection of antineutrophil cytoplasmatic antibodies directed against proteinase 3. Annals of the rheumatic diseases, 69(2), 468-469.

9. Savige, J., Gillis, D., Benson, E., Davies, D., Esnault, V., Falk, R. J., ... \& Wiik, A. (1999). International consensus statement on testing and reporting of antineutrophil cytoplasmic antibodies (ANCA). American journal of clinical pathology, 111(4), 507-513.

10. Vermeersch, P., Vervaeke, S., Blockmans, D., van Hoovels, L., Mariën, G., Vanmaele, H., \& Bossuyt, X. (2008). Determination of antineutrophil cytoplasmic antibodies in small vessel vasculitis: comparative analysis of different strategies. Clinica Chimica Acta, 397(1-2), 77-81.

11. Tervaert, J. W. C., \& Damoiseaux, J. (2012). Antineutrophil cytoplasmic autoantibodies: how are they detected and what is their use for 
diagnosis, classification and follow-up?. Clinical reviews in allergy \& immunology, 43(3), 211-219.

12. Tsiveriotis, K., Tsirogianni, A., Pipi, E., Soufleros, K., \& Papasteriades, C. (2011). Antineutrophil cytoplasmic antibodies testing in a large cohort of unselected greek patients. Autoimmune diseases, 2011:626495.

13. Trevisin, M., Pollock, W., Dimech, W., Melny, J., Paspaliaris, B., Gillis, D., ... \& Savige, J. (2008). Antigen-specific ANCA ELISAs have different sensitivities for active and treated vasculitis and for nonvasculitic disease. American journal of clinical pathology, 129(1), 42-53.

14. Merkel, P. A., Polisson, R. P., Chang, Y., Skates, S. J., \& Niles, J. L. (1997). Prevalence of antineutrophil cytoplasmic antibodies in a large inception cohort of patients with connective tissue disease. Annals of Internal Medicine, 126(11), 866-873.

15. Watts, R., Lane, S., Hanslik, T., Hauser, T., Hellmich, B., Koldingsnes, W., ... \& Scott, D. (2007). Development and validation of a consensus methodology for the classification of the ANCA-associated vasculitides and polyarteritis nodosa for epidemiological studies. Annals of the rheumatic diseases, 66(2), 222-227.

16. Pollock, W., Jovanovich, S., \& Savige, J. (2009). Antineutrophil cytoplasmic antibody (ANCA) testing of routine sera varies in different laboratories but concordance is greater for cytoplasmic fluorescence (C-ANCA) and myeloperoxidase specificity (MPOANCA). Journal of immunological methods, 347(1-2), 19-23.

17. Knütter, I., Hiemann, R., Brumma, T., Büttner, T., Großmann, K., Cusini, M., ... \& Csernok, E. (2012). Automated interpretation of ANCA patterns-a new approach in the serology of ANCA-associated vasculitis. Arthritis research \& therapy, 14(6), 1-11.

18. Hagen, E. C., Daha, M. R., Hermans, J. O., Andrassy, K., Csernok, E., Gaskin, G., ... \& van der Woude, F. J. (1998). Diagnostic value of standardized assays for anti-neutrophil cytoplasmic antibodies in idiopathic systemic vasculitis. Kidney international, 53(3), 743-753.

19. Savige, J., Dimech, W., Fritzler, M., Goeken, J., Hagen, E. C., Jennette, J. C., ... \& International Group for Consensus Statement on Testing and Reporting of Antineutrophil Cytoplasmic Antibodies (ANCA). (2003). Addendum to the International Consensus Statement on testing and reporting of antineutrophil cytoplasmic antibodies: quality control guidelines, comments, and recommendations for testing in other autoimmune diseases. American Journal of Clinical Pathology, 120(3), 312-318. 